\title{
Can superflares occur on the Sun? A view from dynamo theory
}

\author{
M. M. Katsova ${ }^{1 *}$, L. L. Kitchatinov ${ }^{2,3}$, M. A. Livshits ${ }^{4}$, \\ D. L. Moss ${ }^{5}$, D. D. Sokoloff ${ }^{6}$, and I.G. Usoskin ${ }^{7}$
}

\begin{abstract}
Recent data from the Kepler mission has revealed the occurrence of superflares in sun-like stars which exceed by far any observed solar flares in release of energy. Radionuclides data do not provide evidences for occurrence of superflares on the Sun over the past eleven millennia. Stellar data for a subgroup of superflaring Kepler stars are analysed in an attempt to find possible progenitors of their abnormal magnetic activity. A natural idea is that the dynamo mechanism in superflaring stars differs in some respect from that in the Sun. We search for a difference in the dynamo-related parameters between superflaring stars and the Sun to suggest a dynamo-mechanism as close as possible to the conventional solar/stellar dynamo but capable of providing much higher magnetic energy. Dynamo based on joint action of differential rotation and mirror asymmetric motions can in principle result in excitation of two types of magnetic fields. First of all, it is well-known in solar physics dynamo waves. The point is that another magnetic configuration with initial growth and further stabilisation is also possible for excitation. For comparable conditions, magnetic field strength of second configuration is much larger rather of the first one just because dynamo do not spend its efforts for periodic magnetic field inversions but use its for magnetic field growth. We analysed available data from the Kepler mission concerning the superflaring stars in order to find tracers of anomalous magnetic activity. Starting from the recent paper [1]), we find that anti-solar differential rotation or anti-solar sign of the mirrorasymmetry of stellar convection can provide the desired strong magnetic field in dynamo models. We confirm this concept by numerical models of stellar dynamos with corresponding governing parameters. We conclude that the proposed mechanism can plausibly explain the superflaring events at least for some cool stars, including binaries, subgiants and, possibly, low-mass stars and young rapid rotators.
\end{abstract}

\section{Keywords}

Sun: activity, Sun: flares, stars: late-type-stars: activity, dynamo

${ }^{1}$ Sternberg State Astronomical Institute, Lomonosov Moscow State University, 119991, Moscow, Russia maria@sai.msu.ru

2 Institute for Solar-Terrestrial Physics, PO Box 291, Irkutsk, 664033, Russia kit@iszf.irk.ru

${ }^{3}$ Pulkovo Astronomical Observatory, St. Petersburg 196140, Russia

${ }^{4}$ IZMIRAN, 4 Kaluzhskoe Shosse, Troitsk, Moscow, 108840, Russia maliv@mail.ru

${ }^{5}$ School of Mathematics, University of Manchester, Oxford Road, Manchester, M13 9PL, UK david.moss@manchester.ac.uk

${ }^{6}$ Department of Physics, Moscow University, 119992 Moscow, Russia sokoloff.dd@gmail.com

${ }^{7}$ Space Climate Research Unit and Sodankylä Geophysical Observatory, 90014 University of Oulu, Finland ilya.usoskin@oulu.fi 


\section{Contents}

\section{Introduction}

2 A possible origin of superflares

3 The strongest solar flares and related events

4 Dynamo modelling

5 From the classification of superflaring stars to the sign of dynamo number

6 Discussion and conclusions

References

\section{Introduction}

Recent data from the Kepler mission [2] has revealed the existence of stellar "superflares" [3, 4]. Some of the superflaring stars are G-dwarfs with long rotation periods $P_{\text {rot }}>10$ days [4], and two of these stars are very similar to the Sun in their surface temperature and rotation rate [5] Observational techniques reveal flares with total energy substantially greater than $10^{33} \mathrm{erg}$ (to be compared with the highest energy, approximately $10^{32} \mathrm{erg}$, of any observed solar flares - see details in Sect.3) however sometimes the reported energy is as large as $10^{36} \mathrm{erg}$.

Observations of superflares on Sun-like stars are challenging for solar physics in general and for the solar dynamo in particular. On one hand, the energy of the solar magnetic field is insufficient to produce a superflare (assuming a reasonable efficiency of the flare production [6]). On the other hand, cosmogenic isotope studies have identified historical events that were 30-50 times stronger (in the sense of the fluxes of solar energetic particles) than the most energetic solar flares observed instrumentally [3, 7, 8]. It is likely that these events form the upper limit of the intensity of solar events during the last eleven millennia (see Sect.3), while the strongest stellar superflares are substantially stronger.

An important question remains whether superflares with energies up to $10^{35}-10^{36}$ erg are possible on the present-day Sun. If not, what is the physical difference between the Sun and the superflaring Sun-like stars? We stress that this question is beyond purely academic interest because a solar superflare would be hazardous for modern technology. [9] noticed that the dependence of superflare occurrence frequency on the flare energy found in [3] for Kepler targets forms a high-energy continuation of the power-law distribution of the observed solar flares. From this interpolation they suggested an occurrence frequency of flares on the Sun to be one flare with energy $\geq 10^{34}$ erg per 800 years and one flare with energy $\geq 10^{35}$ erg per 5000 years. However, this disagrees with the radionuclide data for the last $\sim 10^{4}$ years $[10,11]$ (see also Section 3) and data from lunar rocks [12]).

\section{A possible origin of superflares}

A step towards addressing the problem was recently undertaken by Kitchatinov \& Olemskoy [1] who suggested a dynamo model based on fluctuations of the dynamo drivers. The fluctuations can drive a solar activity cycle with sufficient magnetic energy to produce flares stronger than flares observed astronomically and similar to those identified in isotopic data. By varying the model parameters even stronger flares can possibly be explained, but hardly the strongest observed stellar flares of the energy of ca. $10^{36}$ erg. 
The key idea of [1] is as follows. The intensity of dynamo drivers can be accumulated in one dimensionless dynamo number $D$

$$
D=\frac{\Delta \Omega \alpha R_{\odot}^{3}}{\eta_{\mathrm{T}}^{2}},
$$

which combines the differential rotation $(\Delta \Omega)$, the eddy diffusivity $\left(\eta_{\mathrm{T}}\right)$ and the rate of toroidal-topoloidal field conversion by cyclonic motions $(\alpha)$ into a single parameter quantifying their common efficiency in producing magnetic activity. With a conventional definition, $D$ is positive for solar-type differential rotation with positive $\alpha$ (in the Northern hemisphere). Note, that reversal of the sign of dynamo number requires either reversal of the sign of $\alpha$ or the sign of $\Delta \Omega$. A reversal of the sign of dynamo number, to become negative, switches the dynamo into a regime with much higher magnetic energy. The expected fluctuations in the dynamo drivers can cause the dynamo number to fluctuate. Sufficiently large fluctuations can produce rare events of sign reversals of $D$ yielding substantially higher magnetic energy and leading, subsequently, to more energetic flares.

The present paper develops this idea further and suggests that Sun-like stars with extremely energetic flares can in fact be different from the Sun. This difference results in dynamo numbers which are regularly negative (rather than positive as in the solar case). The statistics of superflares on Kepler stars does not exclude this possibility. It is found $[4,13]$ that only about $0.3 \%$ of Kepler targets display superflares. Moreover, stars in this small group exhibit a very uneven distribution of flares with $E>10^{34} \mathrm{erg}$, having on average about three superflares per a star per year. Stars of this small group may differ from the bulk of the population of less active stars by hosting dynamos with negative $D$.

Let us present the idea of this paper in a more extended form. Dynamo based on joint action of differential rotation and mirror asymmetric convection (or turbulence) can in principle generate two types of magnetic configuration. First of all, that are conventional for solar physics waves of magnetic field (dynamo waves). The point is another configuration with initial magnetic field growth and then saturation is also possible. This second type of configurations occurs in particular for galactic magnetic fields which do not demonstrate traces of wave-like behaviour. For comparable conditions, magnetic field strength og magnetic fields of the second type is much larger rather for the first one just because abilities of dynamo are not spending on periodic magnetic field reversals rather for magnetic field growth only. Of course, the type of magnetic configurations excited in a particular celestial body depends on the flow structure and geometry of the body however sperical dynamos do demonstrate a region in parametric space with excitation conditions for both types of magnetic fields are close enough to allow excitation of a steady magnetic field instead of an oscillating one.

Paper [1] supposes that a transition from an oscillating to the stationary configuration appears for a limited time as a fluctuation. Here we attract attention to the fact that for many stars (say, for binaries) hydrodynamics may be rather different from the solar one and we can expect generation of stationary magnetic fields. In this case, magnetic field strength can be much larger than the solar one what in turn can results in superflares.

We demonstrate that a dynamo model with negative dynamo number can produce magnetic energy which is several orders of magnitude higher than a similar model with positive dynamo number. We discuss possible physical reasons for stars to have negative dynamo numbers.

An observational and theoretical understanding for the differential rotation of superflaring stars is obviously crucially important for dynamo modelling. Differential rotation of stars observed by Kepler is discussed in [14]. 


\section{The strongest solar flares and related events}

Solar flares have been known since 1859 when a giant white flare was discovered by R. Carrington and R. Hodgson, and were well studied during the 20th and 21st centuries. However, no records of optical flares are known before that. On the other hand, extreme solar events can be studied even for earlier times, using indirect proxy data. Such a proxy, recoverable with stable resolution for about ten millennia backward, is the cosmogenic radionuclide record preserved in a natural terrestrial archive. The most common and useful cosmogenic proxies are ${ }^{14} \mathrm{C}$ (radiocarbon), stored and measured in dendrochronologically dated tree trunks, and ${ }^{10} \mathrm{Be}$, measured in polar ice cores [15]. These cosmogenic proxies are used to reconstruct the flux of galactic cosmic rays (GCR) in the past [16]. Since the GCR flux is modulated by solar/heliospheric magnetic fields, its variability reflects the (inverse) solar activity. While GCR are always present in the vicinity of the Earth and are subject to solar modulation, sporadic events of solar energetic particles (SEPs) can occur sometimes. Such events are related to solar eruptive processes, solar flares or coronal mass ejections, which can accelerate solar particles (mostly protons) to high energies, up to several $\mathrm{GeV}$, which is sufficient to initiate a nucleonic cascade in the Earth's atmosphere and thus to produce cosmogenic nuclides. However, because of the relatively low time-resolution (annual at best) and high level of noise in the data, a SEP event must have high magnitude (an "extreme SEP event") in order to be observable in the proxy record [17].

Presently, only two such extreme SEP events have been discovered in the past. The greatest event around $775 \mathrm{AD}$ was found in high-resolution ${ }^{14} \mathrm{C}$ tree-ring data from Japan [18] and low resolution ${ }^{14} \mathrm{C}$ and ${ }^{10} \mathrm{Be}$ data [19], and later confirmed with other high-resolution ${ }^{14} \mathrm{C}[7,20,21]$ and ${ }^{10} \mathrm{Be}$ and ${ }^{36} \mathrm{Cl}$ records [22]. Although various hypotheses were proposed to explain the event, its solar origin has become a paradigm $[7,8]$. The strength of the $775 \mathrm{AD}$ event was estimated to be about 40 times greater than the strongest SEP event of the instrumental era (23 Feb, 1956). Another similar event was found in the high-resolution ${ }^{14} \mathrm{C}$ data corresponding to the year $994 \mathrm{AD}$ [23], and it is estimated to be a factor 1.5 -2 weaker than that of 775 AD. Interestingly, the Carrington event, which produced a strong white-light flare and the strongest recorded geomagnetic storm [24] was not accompanied by a strong SEP event $[17,19]$.

The SEP event of 775 AD may conservatively serve as the strongest event over the Holocene (the current interglacial period that started about 11 millennia ago). Although only a small fraction of this period is covered by high-resolution annual ${ }^{14} \mathrm{C}$ data, being mostly measured with 5-year resolved (lowresolution) data, it is unlikely that an event stronger than that took place during the Holocene. The team led of the paper [25], which discovered both $775 \mathrm{AD}$ and $994 \mathrm{AD}$ events, has recently performed a systematic search for other events, starting from the high-resolution $\Delta^{14} \mathrm{C}$ measurements around several sharp peaks distinguishable in the low-resolution (decadal average) INTCAL dataset. An analysis of the strongest peak about ( 0.4 permil/year) around $775 \mathrm{AD}$ led to the discovery of the event. This peak was so strong that it was clearly observable even in the low-resolution INTCAL record [19]. Another smaller peak, hardly distinguishable in the low-resolution INTCAL record, corresponded to the discovery of a weaker event in $994 \mathrm{AD}$. However, an analysis of high resolution $\Delta^{14} \mathrm{C}$ measurements around several other peaks $(\approx 0.3$ permil/year) over the last several millennia did not result in finding of new SEP events. Nevertheless there are several relatively strong peaks $(0.3 \div 0.4$ permil/year $)$ in the low resolution INTCAL data over the Holocene, which are not yet covered by high-resolution data. These may potentially contain signals of SEP-like sharp events, but they cannot be much stronger than the event of 775 AD, otherwise they would have been detected in a systematic search over the low-resolution INTCAL dataset [26].

Thus, although we cannot at the moment say that the event of $775 \mathrm{AD}$ was the only of a kind, it can 
be conservatively stated that an event stronger than that did not occur during the Holocene. Accordingly, the event of $775 \mathrm{AD}$ can be considered as the worst case scenario over the last eleven millennia.

We note that the relation between strong flares and extreme SEP events is not one to one. Not every strong flare would produce an SEP event at Earth, depending on the flare's location on the solar disc (flares near the west limb are more effective). This is clearly seen on the example of the Carrington flare which was not accompanied by a strong SEP event. On the other hand, an extreme SEP event should have a parent flare and a strong coronal mass ejection. For example, the greatest directly observed event of $23 \mathrm{Feb}, 1956$ had a strong parent flare of class at least 3-B (it occurred partly behind the west limb and accordingly its intensity might have been underestimated).

Several of the largest solar flares have been observed in the current epoch, such as events in February 1956, August 1972, 1989, October 2003 with total energies close to those of the Carrington flare. Very high-quality data on the total vector of the magnetic field in active regions and the large-scale magnetic field including the dipole field of the Sun as a star are available now. These observations make it possible to calculate the free energy of the magnetic field in active regions that can be released in flares. These estimates show that even the largest active regions on the Sun are capable of producing non-stationary processes (flares and CME) with total energy not greater than $3 \times 10^{32} \mathrm{erg}$. Such an upper limit for a given active region follows from energy considerations, namely from the magnetic virial theorem as well [27]. As for the magnetic fields on active F, G, and $\mathrm{K}$ main-sequence stars, there are modern spectropolarimetric observations indicating that some fast rotating, young sun-like $\mathrm{G}$ stars possess magnetic fields around 5 G [28]. Spots on these stars can cover up to $10 \%$ of a stellar surface. These results, together with the magnetic virial theorem, imply that the maximal possible energy of the strongest flare on these stars cannot exceed $10^{34} \mathrm{erg}$ [29]. Stronger events require for their explanation, either another origin of flares or changes to the dynamo mechanism.

\section{Dynamo modelling}

In order to demonstrate the dependence of the dynamo-generated magnetic energy on the sign of the dynamo number, we use a conventional mean-field model for dynamo in spherical geometry driven by the joint action of the differential rotation and a mirror asymmetric factor $\alpha$ responsible for restoration of the poloidal magnetic field from toroidal (e.g. [30]). The physical nature of the factor $\alpha$ can be associated with the action of magnetic or Coriolis forces. Which of them is more important for stellar dynamos is not crucial for what follows. For the sake of definiteness and simplicity we use the parametrization

$$
\alpha=\frac{\alpha_{0}(r)}{1+\vec{B}^{2}(\vec{r}, t)}
$$

This is the simplest nonlinear suppression of dynamo action, known as algebraic $\alpha$-quenching. In principle, it would not be problematic to include various additional effects, such as meridional circulation and/or more sophisticated types of dynamo quenching. For the sake of definiteness we use two different parametrizations of the stellar rotation curve (Fig. 1), available in literature, viz. a SOHO-like rotation law (e.g. [31]) and the rotation law [32].

The key point of our idea is that the dynamo drivers are not very well-defined quantities and there is a probability that the sign of the main dimensionless number, which determines stellar dynamo action in a Sun-like star, can be opposite to that inferred for the Sun. Physical reasons for such an idea are discussed in the following section while here we consider some consequences of this hypothesis. 


\section{omega}

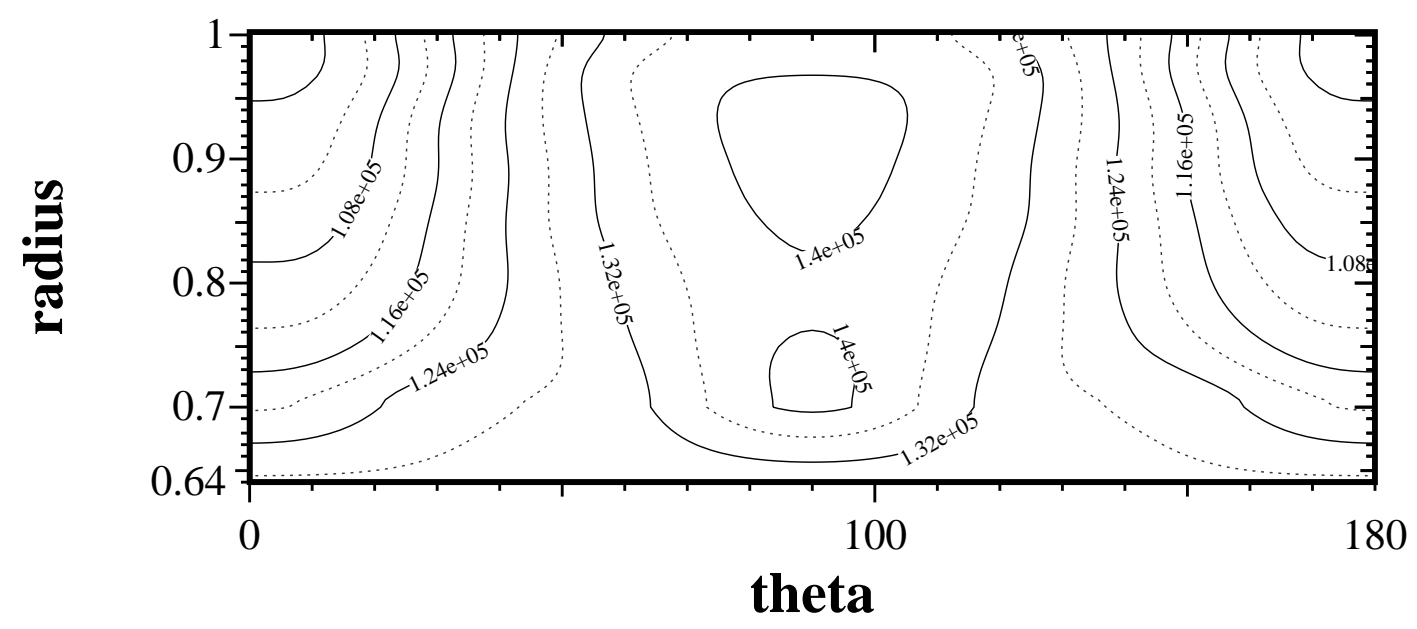

omega

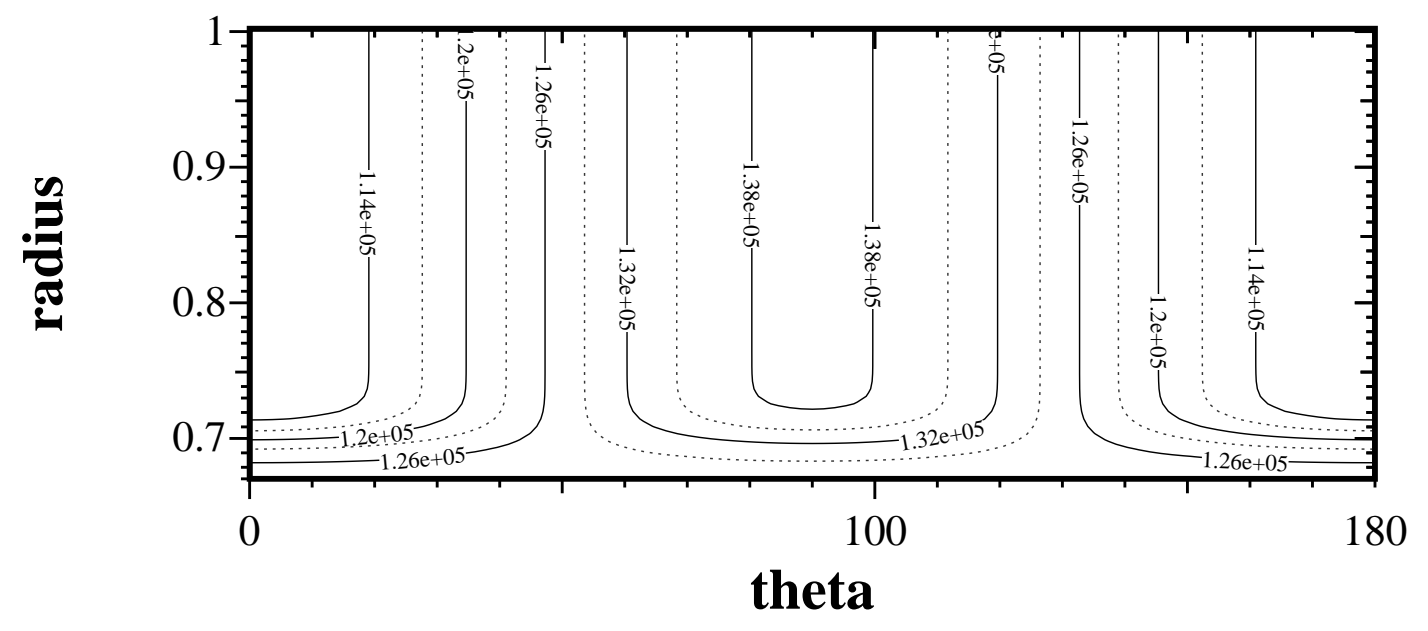

Figure 1. Stellar rotation curves: upper panel-SOHO-like rotation curve, lower panel - [32] rotation curve. 
We run dynamo models with both rotation curves of Fig. 1 and then reverse the sign of $D$ and see what happens with magnetic energy after a steady state is reached. With the SOHO-like rotation curve (Fig. 1, upper panel), the result is quite straightforward. The sign of $D$ determines the direction of dynamo wave propagation so that the activity wave reverses its direction of propagation from equatorward to poleward while the magnetic energy remains more or less the same as before. We do not illustrate this rather standard option in a figure.

The [32] rotation law gives a much more instructive result. Time series for the standard solar case $D>$ 0 are presented in Fig. 2. We conclude that we obtain a standard travelling wave of solar type. A deeper investigation of the result shows that the main direction of dynamo wave propagation is radial, while some projection of the wave vector on the meridional direction provides an equator-ward propagation of activity wave (cf. [33]). For this case the amplitude of $\alpha$ is chosen to be about twice the marginal value for dynamo action.

Reversing the sign of the dynamo number $D$ (Fig. 3) we obtain a steady dynamo producing a magnetic configuration with substantially higher magnetic energy compared with the previous case $(\log E \approx 2.0$ vs. $\log E \approx 0.2$, respectively).

One more instructive point is that the steady ratio between toroidal and poloidal magnetic fields for the magnetic configuration with sign reversed $D$ is about one order of magnitude lower then the average value of this ratio for the oscillating configuration $\left(E_{\mathrm{pol}} / E_{\mathrm{tor}} \approx 4.1 \times 10^{-6}\right.$ to be compared with $6 \times 10^{-4}$ in Figure 2, taking into account that here we deal with quadratic quantities). It means that the strong magnetic field is now more hidden in the stellar interior and it is less observable between the superflares.

\section{From the classification of superflaring stars to the sign of dynamo number}

We expect that hydrodynamics of a particular star with superflares differs somehow from solar hydrodynamics in order to provide a negative dynamo number $D$ what in turn gives a much stronger magnetic field compared to the Sun. Developing a hydrodynamical model for such stars needs to be addressed specifically and it is obviously beyond the scope of this paper. Here we limit ourselves to presenting a list of solar-type stars with superflares from [34] which gives a hint that its hydrodynamic may be substantially different from in the Sun.

Indeed, observations of stellar superflares by the Kepler mission show that energies of most events do not exceed $10^{34} \mathrm{erg}$. However, among sun-like stars with superflares that are characterized in [34] as rotationally variable, there are some objects where more energetic superflares do occur. We try to check whether these stars are solar-type or different. We choose stars with superflares with energies $>10^{35}$ erg, which comprise more than $15 \%$ of the list in [34]. Several examples of such stars are presented in Table 1. An analysis of the properties of the selected stars indicates that the pattern of their variability is significantly different from solar-type activity (see comments to Table 1).

These examples are illustrative only and do not pretend to represent a full choice of possibilities. Additional options include binaries such as the Algol variables (e.g. [39]). Standard models of stellar hydrodynamics used in dynamo studies assume that stars can be considered as single objects (see, however, $[40,41])$. Tidal interactions in stellar convective shells may be associated with non-solar distribution of dynamo drivers. Another possibility here is related to subgiants.

The sign of the dynamo number (see definition of $D$ in Eq. (1) can be opposite to the solar case if either the differential rotation is anti-solar or the sign of $\alpha$ is reversed. Strassmeier [42] reviewed similarities and differences in magnetic activity between cool stars and the Sun. His Table 1 gives several examples 

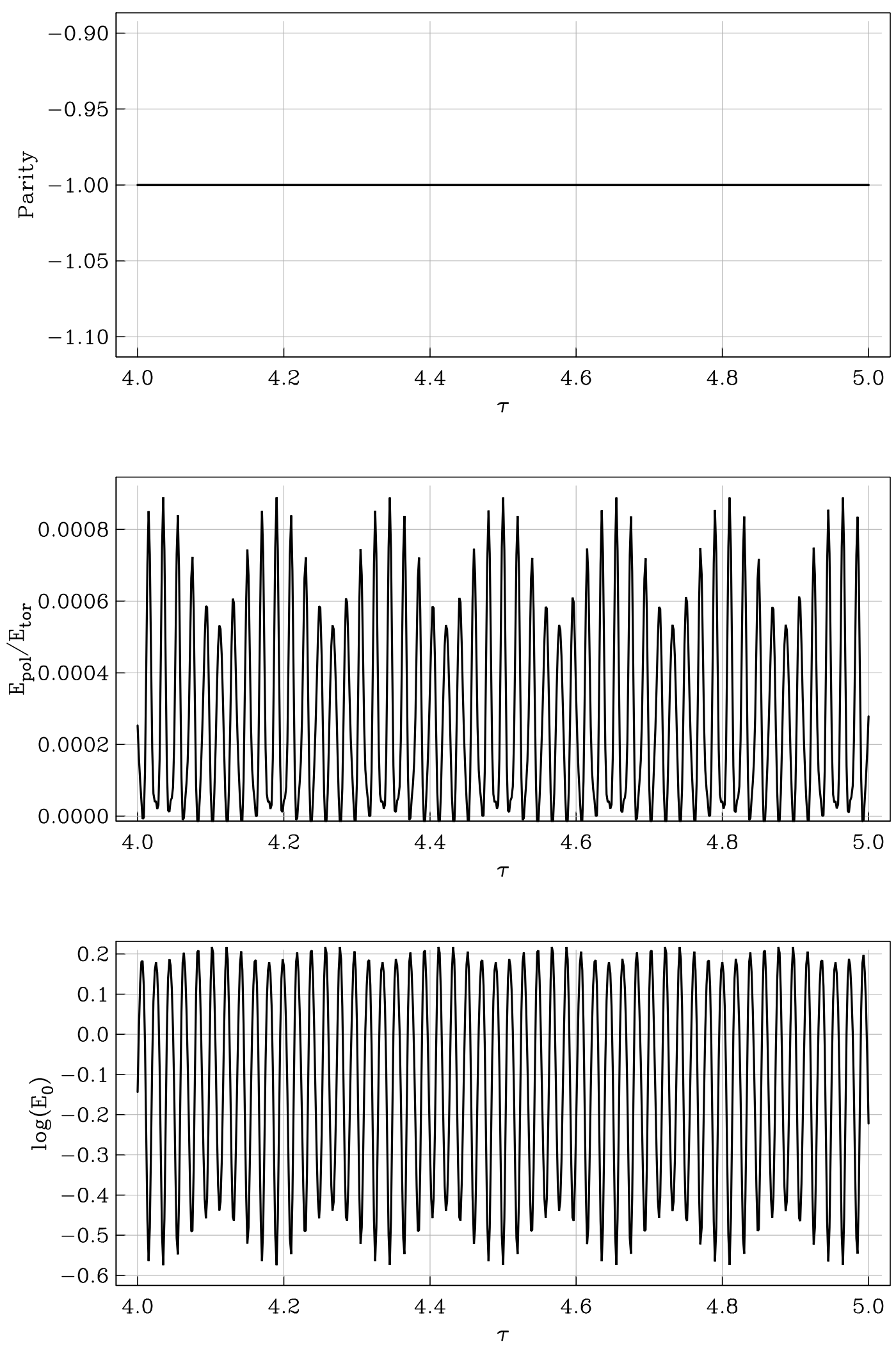

Figure 2. Magnetic field for rotation law [32], D >0, timeseries for parity (top), ratio of magnetic energies of toroidal and poloidal magnetic fields (middle) and total energy of the mean magnetic field (bottom). 

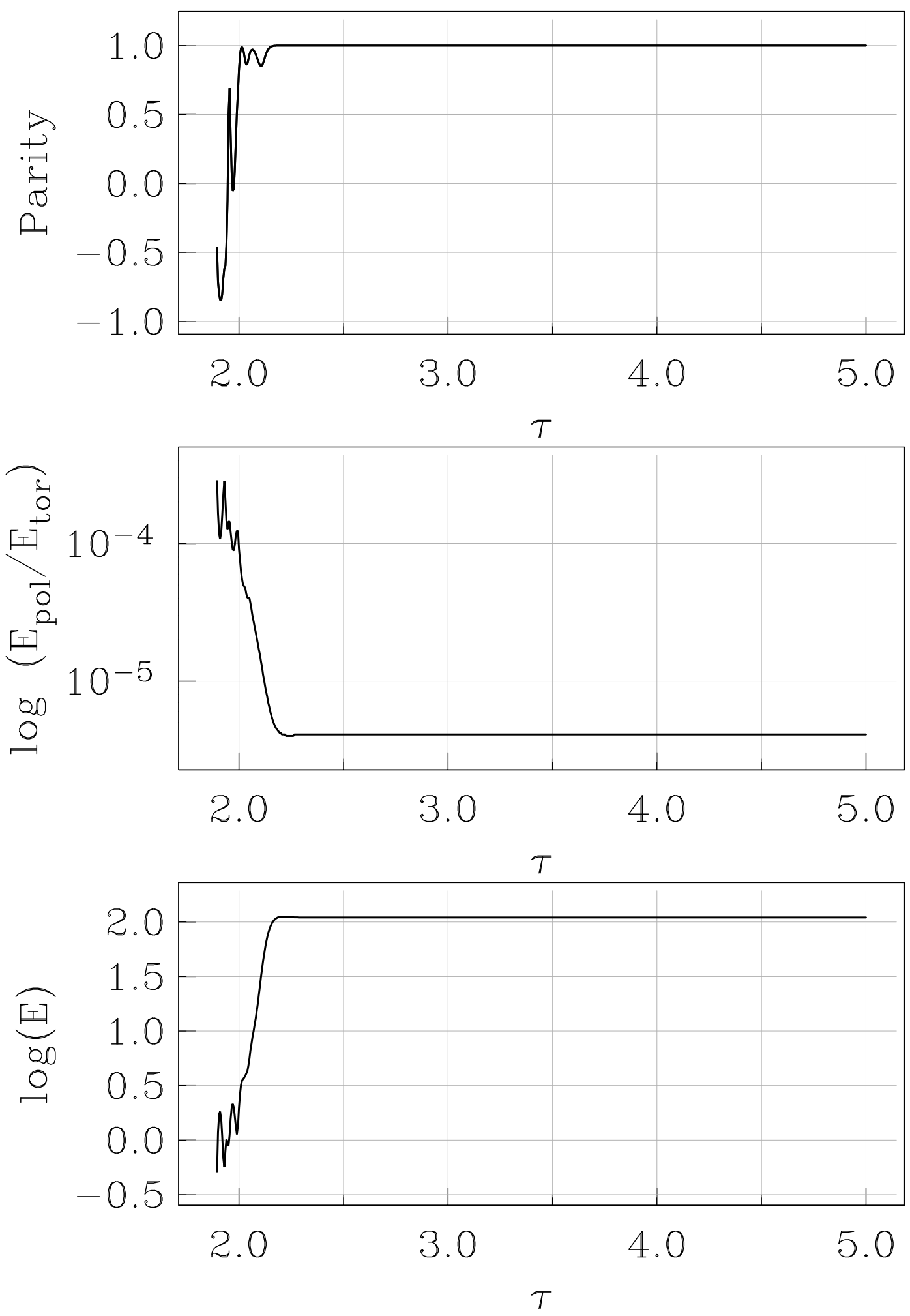

Figure 3. Summary of the fields obtained using the rotation law [32], $D<0$, time series for parity (top), ratio of magnetic energies of toroidal and poloidal magnetic fields (middle) and total energy of the mean magnetic field (bottom). The asymptotic ratio of $E_{\mathrm{pol}} / E_{\mathrm{tor}}$ is approximately $4.1 \times 10^{-6}$. 
Table 1. Some solar-type stars with superflares with $E>10^{35} \mathrm{erg}$, after [34]. $T_{\mathrm{eff}}$ is the effective temperature, $g$ is the gravity in $\mathrm{cm} \mathrm{s}^{-2}, P_{\text {rot }}$ is the rotation period.

\begin{tabular}{|c|c|c|c|c|c|c|}
\hline KIC number & $T_{\text {eff }} \mathrm{K}$, & $\log g$ & $\log E$ & $\mathrm{~N}$ & $P_{\text {rot }}$, day & Comments \\
\hline \multicolumn{7}{|c|}{ Binaries } \\
\hline 1156431 & 5094 & 4.514 & 35.06 & 213 & 3.142 & $\begin{array}{l}\text { Binary/multiple system [35], unusual differential } \\
\text { rotation [36], cooler than the Sun }\end{array}$ \\
\hline 8481574 & 5722 & 4.487 & 35.06 & 2 & 0.326 & eclipsing binary $^{T}$ \\
\hline 9752973 & 5865 & 4.017 & 35.03 & 1 & 13.05 & eclipsing binary $^{\top}$ \\
\hline 12156549 & 5541 & 4.378 & 36.50 & 128 & 3.651 & binary $^{1}$; oscillations in superflares [37] \\
\hline 9655129 & 5140 & 4.431 & 35.38 & 26 & unknown & detatched Algole-type $^{1}$ \\
\hline \multicolumn{7}{|c|}{ Subgiants } \\
\hline 6437385 & 5401 & 3.713 & 36.78 & 18 & 13.672 & oscillations in superflares [37] \\
\hline 7350496 & 5453 & 3.744 & 36.43 & 4 & 9.403 & \\
\hline 8226464 & 5754 & 4.053 & 36.44 & 15 & 3.101 & quasi-periodic pulsations [38] \\
\hline \multicolumn{7}{|c|}{ Stars cooler than the Sun } \\
\hline 3945784 & 4854 & 4.444 & 35.09 & 18 & 15.267 & $\mathrm{~K} 2 \mathrm{~V}$ \\
\hline \multicolumn{7}{|c|}{ Stars with pulsations or oscillations } \\
\hline 5475645 & 5336 & 4.654 & 35.63 & 6 & 7.452 & quasi-poeriodic pulsations [37] \\
\hline 11610797 & 5865 & 4.465 & 35.76 & 34 & 1.625 & oscillations in superflares [37] \\
\hline \multicolumn{7}{|c|}{ Very young fast rotating stars } \\
\hline 9652680 & 5618 & 4.802 & 35.38 & 26 & 1.408 & \\
\hline
\end{tabular}

${ }^{1}$ Information concerning stellar variability is added from the SIMBAD database, provided by CDS, Strasbourg.

of observed anti-solar rotation. All of them belong to close binaries or giants. For MHD modelling of the transition from solar to anti-solar rotation see [43].

Less evident options are sun-like stars substantially cooler than the Sun, very young stars and stars which demonstrate various pulsations and oscillations. The trend for superflare activity to increase with decreasing temperature was established and explained in [13] (see also [44]). Rapidly rotating young stars may have negative $\alpha$ because of strong twisting of rising magnetic loops by the Coriolis force [45], so the young Sun probably had superflares. Differential rotation in young rapidly rotating stars was recently observed $[46,47]$. For slower rotating sun- like F, G, and K stars, discovered during HK Project, differential rotation here has been investigated in [48].

\section{Discussion and conclusions}

In this paper we suggest a scenario which allows us to understand how a stellar dynamo can provide a solar-like star with a magnetic field whose energy is substantially larger than that of the Sun. This in turn can explain why superflares of $\gtrsim 10^{35} \mathrm{erg}$ can occur on solar-type stars - and some stars emanate them several times in a limited time interval - while radionuclide data do not provide any evidences for solar flares of comparable energy over the past eleven millennia.

Our scenario originates from the idea of [1], and suggests that the sign of the dynamo drivers in the superflaring stars can be opposite to that of the sun on a regular basis rather than reverse occasionally as a result of rare fluctuations. Stellar dynamo simulations for stars with differential rotation dependent mainly on latitude show a change from oscillatory to steady dynamo action with a strong increase in magnetic energy when the sign of the dynamo number is reversed. The sign reversal can be associated 
with anti-solar differential rotation or, perhaps, with change of sign of the $\alpha$-parameter compared to that of the Sun.

We present arguments that the deviations of stellar hydrodynamics from the solar one which can provide the intensive magnetic field can happen at least in some of the stars with superflares. The main straightforward reason for such a deviation can be the fact that some superflaring stars belong to binary systems. Observations favour anti-solar rotation in close binaries [42]. Other examples of superflaring stars include giants, low mass main sequence dwarfs or young rapid rotators.

At this stage of research it looks too early to insist that the scenario suggested can explain all cases of stellar superflares, however it can explain at least some of them. Of course, the scenario explains only how to get high magnetic energy, while the detailed mechanism of flare production requires more than just dynamo studies. Some discussion concerning such processes can be found in, e.g., [29, 49].

\section{Acknowledgments}

DS acknowledges financial support from RFBR under grant 15-02-01407. IU's contribution was made in the framework of ReSoLVE Centre of Excellence (Academy of Finland, project no. 272157). LLK is thankful to the Russian Foundation for Basic Research for the support (project 17-02-00016). MMK is grateful to the RFBR (grant 15-02-06271) and the Leading Scientific Schools project 9570.2016.2 for financial support. We are grateful to referees for useful comments.

\section{References}

[1] Kitchatinov, L. L., \& Olemskoy, S. V. 2016, MNRAS, 459, 4353

[2] Koch D. G., Borucki, W. J., Basri, G. et al. 2010, ApJ, 713, L79

[3] Maehara, H., Shibayama, T., Notsu, S. et al. 2012, Nature, 485, 478

[4] Shibayama, T., Maehara, H., Notsu, S. et al. 2013, ApJS, 209, 5

[5] Nogami, D., Notsu Y., Honda S. et al. 2014, PASJ, 66, L4

[6] Aulanier, G., Démoulin, P., Schrijver, C. J. et al. 2013, A\&A, 549, A66

[7] Usoskin, I. G., Kromer, B., Ludlow, F. et al. 2013, A\&A, 552, L3

[8] Mekhaldi, F., Muscheler, R., Adolphi, F. et al. 2015, Nature Comm., 6, 8611

[9] Shibata, K., Isobe, H., Hiller, A. et al. 2013, PASJ, 65, 49

[10] Hudson, H. S. 2010, Nature Phys., 6, 637

[11] Schrijver, C. J. , Beer, J., Baltensperger, U. et al. 2012, J. Geophys. Res., 117, A08103

[12] Kovaltsov, G.A., Usoskin, I.G. 2014, Solar Phys., 289, 211

[13] Candelaresi, S., Hillier, A., Maehara, H., Brandenburg, A., \& Shibata, K. 2014, ApJ, 792, 67

[14] Balona, L.A., Abedigamba, O.P., 2016, MNRAS, 461, 497

[15] Beer, J., McCracken, K., \& von Steiger, R. 2012, Cosmogenic Radionuclides: Theory and applications in the Terrestrial and Space Enviroments (Berlin: Springer)

[16] Usoskin, I. G. 2017, Living Rev. Sol. Phys. 14, 3

[17] Usoskin, I. G., Solanki, S. K., Kovaltsov, G. A., Beer, J., \& Kromer, B. 2006, Geophys. Res. Lett., 33, 8107 
[18] Miyake, F., Nagaya, K., Masuda, K., \& Nakamura, T. 2012, Nature, 486, 240

[19] Usoskin, I. G., \& Kovaltsov, G. A. 2012, ApJ, 757, 92

[20] Jull, A. J. T., Panyushkina, I. P., Lange, T. E. et al. 2014, Geophys. Res. Lett., 41, 3004

[21] Güttler, D., Adolphi, F., Beer, J. et al. 2015, Earth Planet. Sci. Lett., 411, 290

[22] Sigl, M., Winstrup, M., McConnell, J. R. et al. 2015, Nature, 523, 543

[23] Miyake, F., Masuda, K., \& Nakamura, T. 2013, Nature Comm., 4, 1748

[24] Cliver, E. W., Dietrich, W. F. 2013, J. Space Weath. Space Clim., 3, A31

[25] Miyake,F., Jull, A.J.T., Panyushkina, I.P., Wacker, L., Salzer, M., Baisan, C.H.; Lange, T., Cruz, R., Masuda, K., Nakamura, T. 2017, Proc. Nat. Acad. Sci., 114, 881

[26] Sukhodolov, T., Usoskin, I, Rozanov, E. et al., 2017, Sci. Rep. (in press)

[27] Livshits, M.A., Rudenko, G.V., Katsova, M.M., Myshyakov, I.I. 2015, Adv.Space Res., 55,920

[28] Marsden, S.C., Petit, P., Jeffers, S.V., Morin, J., Fares, R., Reiners, A., do Nascimento, J.D., Auriére, M., Bouvier, J., Carter, B.D., Catala, C., Dintrans, B., Donati, J.-F., Gastine, T., Jardine, M.; Konstantinova-Antova, R., Lanoux, J., Lignieres, F., Morgenthaler, A., RamirezVélez, J.C., Théado, S., Van Grootel, V., BCool Collaboration, 2014, MNRAS, 444, 3517

[29] Katsova M. M., Livshits M. A. 2015, Solar Phys., 290, 3663

[30] Krause, F., \& Rädler, K.-H. 1980, Mean-Field Magnetohydrodynamics and Dynamo Theory (Akademie-Verlag, Berlin)

[31] Moss, D. 1999, MNRAS, 306, 300

[32] Jouve L., Brun, A. S., Arlt, R. et al. 2008, A\&A, 483, 949

[33] Kitchatinov, L. L. 2002, Astronomy Letters, 28, 626

[34] Balona, L.A, 2015, MNRAS, 447, 2714

[35] Reinhold T., Reiners A., Basri G., 2013, A\&A, 560, A4

[36] Balona, L.A., Broomhall, A.-M., Kosovichev, A., Nakariakov, V.M., Pugh, C.E., Doorsselaere, T., 2015, MNRAS, 450, 956

[37] Pugh C.E., Armstrong D.J., Nakariakov V.M., Broomhall A.-M., 2016, MNRAS, 459, 3659

[38] Baize, P., 1954, Mesures d'etoile doubles faites á l'Observatoire de Paria Journal des Observateures, 37, 73

[39] Chen, W.-C., Li, X.-D., Qian, S.-B., 2006, ApJ, 649, 973

[40] Moss, D., Tuominen, I. 1998, A\&A, 321, 151

[41] Moss, D., Piskunov, N., \& Sokoloff, D. 2002, A\&A, 396, 885

[42] Strassmeier, K G. 2004, in Stars as Suns: Activity, Evolution and Planets, Proc. IAUS 219, eds. A. K. Dupree and A. O. Benz (San Francisco, ASP), 11-27

[43] Karak, B. B., Käpylä, P. J., Käpylä, M. J., Brandenburg, A., Olspert N., Pelt J. 2015, A\&A, 576, A26

[44] Maehara, H., Notsu, Y., Notsu, S., Namekata, K., Honda, S., Ishii, T.T., Nogami, D., Shibata, K., 2017, arXiv:1702.07141

[45] Kitchatinov, L. L., \& Olemskoy, S. V. 2015, RAA, 15, 1801 
[46] Distefano, E., Lanzafame, A.C., Lanza, A.F., Messina, S., Spada, F., 2016, A\&A, 591, A43.

[47] Nagel, E., Czesla, S., Schmitt, J.H.M.M., 2016, A\&A, 590, A47.

[48] Katsova, M. M., Livshits, M. A., Soon, W., Baliunas, S. L., Sokoloff, D. D. 2010, New Astronomy, 15, 274

[49] Katsova, M. M., Livshits, M. A., Mishenina, T. V., Nizamov, B. A. 2016, 19th Cambridge Workshop on Cool Stars, Stellar Systems, and the Sun, id. 124 